\title{
Calibración y validación del modelo hidrológico SWMM en cuencas hidrográficas de alta pendiente en Costa Rica
}

\author{
Calibration and validation of the SWMM hydrological \\ model on steep terrain watersheds in Costa Rica.
}

Maikel Méndez-Morales'

Fecha de recepción: 3 de setiembre del 2012

Fecha de aprobación: 17 de diciembre del 2012

Méndez-Morales, M. Calibración y validación del modelo hidrológico SWMM en cuencas hidrográficas de alta pendiente en Costa Rica. Tecnología en Marcha. Vol. 26, N 2. Pág 20-32 


\section{Palabras clave}

SIG; hidrología; modelación; PEST, SWMM.

\section{Resumen}

Se aplicó el modelo hidrológico SWMM en las cuencas experimentales del río Toyogres y la quebrada Zopilote en Cartago, Costa Rica. En la calibración/análisis de sensibilidad del modelo se utilizó el software PEST. La parametrización inicial se fundamentó en información espacial de alta resolución. En el proceso de calibración/validación se utilizaron 13 tormentas registradas entre septiembre y noviembre de 20 I I. Los resultados muestran una eficiencia NS media de $0.85 \pm 0.07$ en calibración y $0.87 \pm 0.04$ en validación para la cuenca del Toyogres, mientras que para el Zopilote, estos valores alcanzan $0.80 \pm$ 0.04 y $0.77 \pm 0.0$ I respectivamente.

Los coeficientes de rugosidad en flujo superficial sobre el área impermeable (nimp), flujo superficial sobre el área permeable (nper) y tránsito hidráulico (ncha) fueron los parámetros más sensibles. Su comportamiento resultó ser parcialmente dependiente de la intensidad y el volumen total de precipitación. Igualmente, existe una profunda dependencia con respecto al estado de humedad antecedente del suelo y los estados de flujo hidráulico, tanto a nivel de superficie como de red de drenaje.

Esto indica que no existe un único set óptimo de parámetros que se adapte satisfactoriamente a todas las condiciones. Aun cuando los coeficientes de variación de los parámetros son relativamente altos, SWMM demostró ser lo suficientemente flexible como para ser utilizado en condiciones donde no existen datos de calibración/validación.

\section{Key words}

GIS; hydrology; modeling; PEST; SWMM

\section{Abstract}

The hydrologic model SWMM was applied in the experimental watersheds of the Toyogres and Zopilote rivers in Costa Rica. The suite PEST was used for model's parameter optimization and sensitivity analysis. Initial parameterization of the model was based on high-resolution spatial information. In the calibration/validation process, 13 rainstorms, recorded between September and November 20 I I, were used. Results show an average NS efficiency of $0.85 \pm 0.07$ in calibration and $0.87 \pm 0.04$ in validation for the Toyogres River, whereas $0.80 \pm 0.04$ and 0.77 \pm 0.01 were respectively found for the Zopilote River.The Manning's roughness coefficient for runoff generation over impervious area (nimp), runoff generation over pervious area (nper) and hydraulic transit (ncha) were the most sensitive parameters of them all.

Their behavior is partially dependent on total volume and rainfall intensity. Furthermore, there is a profound dependency with the antecedent soil moisture conditions and the state of the hydraulic flow in both, surface runoff generation and drainage network.

It is concluded that there is no unique set of optimum parameters capable to satisfy every possible condition. Even when the coefficients of variation of the sensitive parameters are relatively high, SWMM has shown sufficient flexibility to be used even in conditions where three is no calibration/validation data. 


\section{Introducción}

Las grandes áreas metropolitanas se caracterizan por un continuo incremento en su nivel de impermeabilidad, causado principalmente por el desarrollo urbanístico. Con el incremento de la impermeabilidad, aumentan igualmente los volúmenes de escorrentía, los caudales pico y la concentración de contaminantes en las redes de drenajes, lo cual acarrea consecuencias negativas para los sistemas naturales (Barco et al., 2008).

Los modelos hidrológicos distribuidos de precipitación-escorrentía, físicamente basados, representan una herramienta poderosa en el análisis detallado, la simulación y la predicción del comportamiento de los sistemas urbanos de drenaje, amparados principalmente en información espacial proveniente de los Sistemas de Información Geográfica (SIG) (Boyko y Treebushny, 2006). Este tipo de modelos se aplica primordialmente al estudio de eventos de tormentas, con lo cual es posible analizar el impacto hidrológico que estas tienen sobre procesos tales como inundaciones, transporte de sedimentos, transporte de contaminantes e hidráulica de alcantarillados (Hsu et al., 2000, Marcel van der Perk, 2003, Veihe et al., 200 I). Los modelos distribuidos, cuyos parámetros poseen un inherente sentido físico, representan el centro de investigación de la modelación hidrológica, en el tanto en que estos modelos son menos dependientes de procesos de calibración, lo cual los hace particularmente útiles en cuencas no instrumentadas. Además, la interpretatividad de los parámetros físicos arroja una idea clara del comportamiento hidrológico de una cuenca (Huber y Dickinson 1988, Jain et al., 2004, Maneta et al., 2007).

Dado que los modelos no son más que aproximaciones de sistemas reales, la confiabilidad de sus predicciones dependerá de qué tan bien haya sido definida la estructura matemática de estos y qué tan bien haya sido parametrizado (Hogue et al., 2006). Por otro lado, para que un modelo se considere confiable, debe ser capaz de predecir el comportamiento del sistema real bajo una amplia gama de condiciones sobre un periodo extendido de tiempo. Lo anterior puede lograrse mediante la calibración secuencial del modelo.

La calibración de un modelo se alcanza mediante la optimización de los parámetros envueltos en su estructura matemática, comparando cualitativa y cuantitativamente la respuesta del modelo con una serie de mediciones de campo u observaciones, razón por la cual se le denomina un proceso inverso (Gupta y Sorooshian, 1985). En el caso de la hidrología, las observaciones ligadas a los registros temporales de flujo representan la mayor fuente de información sobre el grado de respuesta de la cuenca ante un determinado evento de precipitación. Tras el proceso inverso de calibración de un modelo, el set óptimo de parámetros encontrado define el mínimo absoluto del espacio paramétrico del modelo (Gallagher y Doherty, 2007). Un aspecto clave es determinar si este mínimo absoluto varía de acuerdo con los diversos sets óptimos de parámetros de forma predecible, en virtud de las condiciones de frontera y el estado propio del sistema en un determinado momento. Se ha encontrado dependencia de los parámetros de varios modelos hidrológicos distribuidos con respecto al grado de humedad antecedente del suelo, así como de la intensidad y distribución de las tormentas.

Este trabajo pretende implementar el modelo hidrológico totalmente distribuido SWMM en dos cuencas experimentales de alta pendiente, utilizando información espacial y temporal de alta resolución. Se desea, a su vez, evaluar la variabilidad espacial y temporal de los parámetros que dominan el comportamiento del modelo y determinar si existe un único set óptimo de parámetros.

\section{Metodología}

Área de estudio

El área de estudio se ubica al noroeste de la provincia de Cartago, Costa Rica, e incluye las cuencas del río Toyogres y la quebrada Zopilote. Ambas cuencas, con un área de $14,6 \mathrm{~km}^{2}$, drenan sus aguas en el río Agua Caliente. El área se extiende desde los 9.7 hasta los 10.1 grados de latitud norte y entre los 84.0 y 83.7 grados de longitud oeste. El uso del suelo contempla primordialmente áreas de cultivo y uso urbano en la parte sur. La condición de terreno es abrupta, con un cambio de elevación en el orden de los 980 msnl, desde las cercanías del volcán Irazú (2260 msnl) hasta el centro urbano de Cartago ( $280 \mathrm{msnl})$. 


\section{Descripción del modelo SWMM}

El SWMM (siglas en inglés de Storm Water Management Model) es un modelo físicamente basado totalmente distribuido para el análisis hidrológico e hidráulico de redes de drenaje esencialmente urbanas (Huber y Dickinson 1988). Estructuralmente, el SWMM simula la respuesta de la cuenca a partir de datos de precipitación y parámetros físicos y numéricos relevantes. El modelo conceptualiza los diversos procesos dentro del dominio del balance hídrico a manera de compartimentos. La conceptualización del modelo se basa en entidades de tipo vectorial, líneas (cauces) y nudos (pozos de registros, cambios de pendientes) y polígonos (microcuencas o áreas tributarias), lo cual resume el sistema de drenaje a una estructura de red dendrítica. Una vez que el SWMM genera la respuesta de la cuenca a un determinado evento de precipitación, esta se expresa a manera de hidrograma. El hidrograma representa la evolución temporal de la tasa de flujo en el punto de descarga de la cuenca. No obstante, esta respuesta debe ser calibrada contra registros reales de flujo.

El SWMM utiliza $n$ modelo de onda cinemática basado en los planteamientos de Chezy-Manning para describir el movimiento y propagación hidráulica de los flujos generados por escorrentía superficial:

$\mathrm{Q}=\frac{\mathrm{W}(\mathrm{d}-\mathrm{dp})^{5 / 3} \mathrm{So}^{1 / 2}}{\eta}$

Donde W representa el ancho característico del plano del modelo cinemático, [L]; d es la profundidad de agua en el reservorio, [L]; So es la pendiente promedio de la cuenca, [-]; $\eta$ es el coeficiente de rugosidad de Manning para la superficie, [t L-I/3] y $\mathrm{Q}$ es la tasa de flujo, [L $\mathrm{t}-\mathrm{I}]$.

Para efectos de este estudio, la mayoría de los parámetros del modelo SWMM (tanto medibles como inferibles) fueron derivados a través de las capas de información geográfica disponibles y se resumen a: área (area), pendiente (slope), porcentaje de área impermeable (pimp), número de curva SCS-CN (cn), ancho característico del plano del modelo cinemático (widt), láminas de almacenamiento en depresión sobre el área impermeable (dimp) y sobre el área permeable (dper), coeficientes de rugosidad de Manning tanto para el flujo superficial sobre el área impermeable (nimp), flujo superficial sobre el área permeable (nper) y tránsito hidráulico (ncha).

Calibración y análisis de sensibilidad

Dado que la selección y posterior optimización manual de parámetros medibles o inferibles es por demás subjetiva, resulta difícil evaluar la confiabilidad de las predicciones de tal modelo (Kumar et al., 2009). Para calibrar y validar el modelo SWMM, se utilizó el paquete de optimización automática de parámetros PEST (Doherty, 2005). PEST es un programa de búsqueda basado en los algoritmos de Gauss-Marquardt-Levenberg (GML), el cual examina el espacio paramétrico del modelo con el objeto de encontrar el set de parámetros óptimo que minimice las desviaciones entre observaciones y modelaciones. PEST utiliza la suma del cuadrado de las desviaciones $\mathrm{PHI}(\Phi)$ como su función objetivo (Skahill, 2009), la cual se puede expresar matemáticamente como:

$$
\varphi=\sum_{\mathrm{i}=1}^{\mathrm{n}} \mathrm{w}_{\mathrm{i}}\left(\mathrm{O}_{\mathrm{i}}-\mathrm{M}_{\mathrm{i}}\right)^{2}
$$

Donde $\mathrm{n}$ representa el número total de observaciones, [-]; Oi es el valor observado en el intervalo i, [-]; Mi es el valor modelado en el intervalo i, [-] y wi es el peso relativo asignado a cada observación [-].

Al inicio de cada iteración, PEST varía cada parámetro ajustable de forma incremental y reejecuta el modelo con el propósito de evaluar su sensibilidad. La sensibilidad de cada parámetro se calcula utilizando una forma normalizada de una matriz Jacobiana (J), la cual retiene la derivada parcial de cada parámetro. La sensibilidad compuesta (s) de cada parámetro (I) es la magnitud del vector compuesto por la columna de la matriz Jacobiana perteneciente al parámetro correspondiente y multiplicado por el peso relativo asignado a cada observación almacenada en la matriz cuadrada diagonal (Q) y dividida por el número total de observaciones $(\boldsymbol{m})$ de acuerdo con lo estipulado a continuación:

$\mathrm{S}_{\mathrm{L}}=\left(\mathrm{J}^{\mathrm{t}} \mathrm{QJ}\right)^{1 / 2} \mathrm{LL} / \mathrm{m}$ 
En términos de evaluación de la calidad del proceso de calibración con PEST, se seleccionó la función objetivo de eficiencia de Nash y Sutcliffe (1970), la cual está definida como:

$$
\mathrm{NS}=1-\frac{\sum_{\mathrm{i}=1}^{\mathrm{n}}\left(\mathrm{Q}_{\mathrm{obs}_{\mathrm{i}}}-\mathrm{Q}_{\mathrm{m}_{\mathrm{i}}}\right)^{2}}{\sum_{\mathrm{i}=1}^{\mathrm{n}}\left(\mathrm{Q}_{\mathrm{obs}_{\mathrm{i}}}-\overline{\mathrm{Q}}_{\mathrm{obs}}\right)^{2}} \ldots \ldots \ldots
$$

Donde NS representa la eficiencia en términos fraccionarios, [-]; Qobsi representa las observaciones de caudal, [L3/T]; Qmi es el valor modelado del caudal, $[\mathrm{L} 3 / T]$ y Qobs representa el promedio de las observaciones de caudal, [L3/T].

El error relativo absoluto se utilizó en la cuantificación del error volumétrico relativo (Ev); el error pico relativo (Ep) y el error relativo al tiempo pico (Ept). La ecuación general está definida como:

$$
\text { Error }=1-\frac{\left|\sum_{i=1}^{n}\left(O_{i}-M_{i}\right)\right|}{\sum_{i=1}^{n}\left(O_{i}\right)}
$$

Donde $n$ representa el número total de observaciones, [-]; Oi es el valor observado en el intervalo i, [-] y $\mathrm{Mi}$ es el valor modelado en el intervalo $\mathrm{i}$.

\section{Datos de entrada}

En el proceso de optimización de SWMM se utilizaron 13 eventos de tormenta, ocurridos entre septiembre y noviembre de 2011. De las 13 tormentas, 10 se utilizaron en calibración y 3 en validación. Tales tormentas fueron registradas por tres estaciones meteorológicas instaladas en sitios estratégicos del área de estudio (figura |A). Respecto a la medición del flujo y posterior construcción de los hidrogramas observados, se instalaron 4 sensores en los cauces del río Toyogres y la quebrada Zopilote (figura IA).

\section{Configuración de PEST}

Para la calibración de SWMM con PEST (cuadro I), se eligieron ocho grupos de parámetros, cuya respuesta determina el tamaño, la extensión y la geometría de los hidrogramas.

Con el objeto de disminuir el número de incógnitas y mejorar la confiabilidad sobre las predicciones del modelo, se procedió a restringir el espacio paramétrico de SWMM definiendo grupos de

Cuadro I. Grupos de parámetros de SWMM elegidos para la calibración con PEST.

\begin{tabular}{|l|c|c|c|c|c|c|}
\hline \multicolumn{1}{|c|}{ Parametro } & Nomenclatura & Transformación & Carácter & $\begin{array}{c}\text { Valor } \\
\text { inicial }\end{array}$ & $\begin{array}{c}\text { Valor } \\
\text { mínimo }\end{array}$ \\
máximo
\end{tabular}

Huber y Dickinson (1988)

Tsihrintzis y Hamis (1998)

Wanielista y Yousef (1993)

Chow et al. (1988)

Mays (200I) 
parámetros "agregados" y "distribuidos". Los parámetros agregados se consideran espacialmente homogéneos, por lo que comparten el mismo valor independientemente de su posición geográfica. Los parámetros distribuidos, por su parte, se consideran no homogéneos, por lo que su valor varía a nivel de microcuenca.

\section{Resultados y analisis}

\section{Discretización espacial}

La Figura IA muestra la extensión de las dos cuencas en estudio. Como logra notarse (Cuadro 2), la cuenca de la quebrada Zopilote (239 ha) es apenas una fracción del área correspondiente al río Toyogres ( 220 ha), razón por la cual se utilizaron diferentes umbrales de acumulación en ambas cuencas. La Figura IB ilustra la extensión de las redes de drenaje y la distribución de microcuencas por cuenca.

La alargada geometría en ambas cuencas, explicada a partir del Índice de Forma (IF), el cual representa el cociente entre ancho y largo de una cuenca o área tributaria (cuadro 2), evidencia una geometría muy alargada, lo cual promueve tiempos de concentración muy cortos y consecuentemente una rápida reactividad hidrológica en las microcuencas. Otro factor que contribuye de manera importante a esta situación es la pronunciada condición de pendiente imperante en la zona, imponiéndose un promedio de $24.27 \% \pm 6.45$ como CV para la cuenca del río Toyogres y $16.93 \% \pm 7.14$ para el caso de la quebrada Zopilote. Los porcentajes de área impermeable son considerablemente altos en ambas cuencas, por arriba del 20\% (cuadro 2).

\section{Calibración de tormentas}

Todos los eventos de tormenta estudiados fueron capaces de producir una respuesta en los puntos de concentración ligados a los sensores de flujo. Los cuadros 3 y 4 listan las tormentas analizadas por fecha, junto con su máxima intensidad en $\mathrm{mm} /$ $\mathrm{hr} / \mathrm{min}$ y su volumen total precipitado en $\mathrm{mm}$ para ambas cuencas, donde $V$, representa el volumen escurrido; P, el caudal pico; Pt, el tiempo pico; NS, la eficiencia de Nash Sutcliffe; Ev, el error volumétrico

Figura I. Delimitación, red de drenaje, microcuencas para la cuenca del río Toyogres y la quebrada Zopilote.

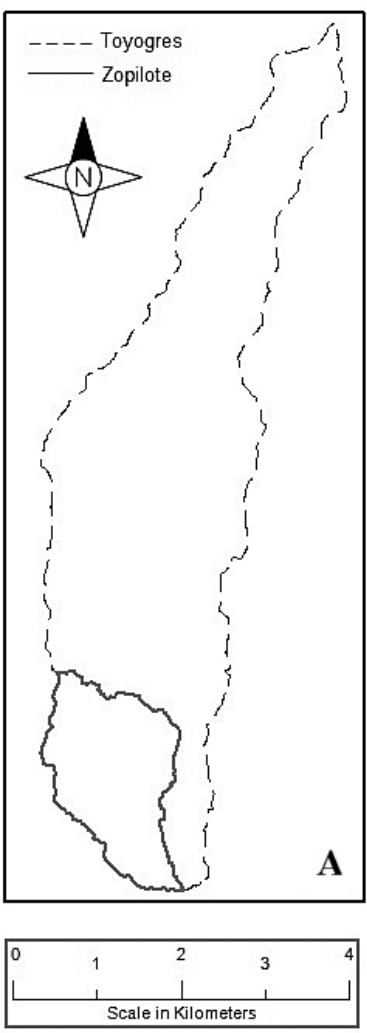

Cuadro 2. Parámetros agregados para la cuenca del Río Toyogres y la micro cuenca de la Quebrada Zopilote.

\begin{tabular}{|c|c|c|c|c|c|c|c|c|c|}
\hline \multirow[b]{2}{*}{$\begin{array}{c}\text { ID } \\
\text { Cuenca }\end{array}$} & \multirow[b]{2}{*}{$\begin{array}{l}\text { Área } \\
\text { (ha) }\end{array}$} & \multirow[b]{2}{*}{$\begin{array}{l}\text { Porcentaje de } \\
\text { área imperme- } \\
\text { able }(\%)\end{array}$} & \multirow{2}{*}{$\begin{array}{c}\text { Ancho } \\
\text { del plano } \\
\text { cinemático } \\
(\mathrm{m})\end{array}$} & \multirow[b]{2}{*}{$\begin{array}{l}\text { Indice del } \\
\text { forma (-) }\end{array}$} & \multirow[b]{2}{*}{$\begin{array}{c}\text { Pendiente } \\
(\%)\end{array}$} & \multirow[b]{2}{*}{$\begin{array}{c}\text { Número de } \\
\text { curva SCS- } \\
\text { CN }\end{array}$} & \multicolumn{3}{|c|}{ Posición del centroide (CRTM05) } \\
\hline & & & & & & & Este (m) & Norte (m) & $\begin{array}{c}\text { Elevación } \\
\text { (msnm) }\end{array}$ \\
\hline Toyogres & 1219.83 & $27.18 \pm 16.62$ & $\begin{array}{c}4160.46 \pm \\
1887.23\end{array}$ & $0.12 \pm 0.06$ & $24.27 \pm 6.45$ & $69.50 \pm 5.24$ & 509950.00 & 1092391.00 & 1356.65 \\
\hline $\begin{array}{l}\text { Zopi- } \\
\text { lote }\end{array}$ & 237.80 & $23.61 \pm 13.48$ & $\begin{array}{c}1381.10 \pm \\
773.10\end{array}$ & $\begin{array}{c}0.21 \pm \\
0.26\end{array}$ & $16.93 \pm 7.14$ & $69.42 \pm 5.3$ & 509556.00 & 1088766.00 & 1120.58 \\
\hline
\end{tabular}




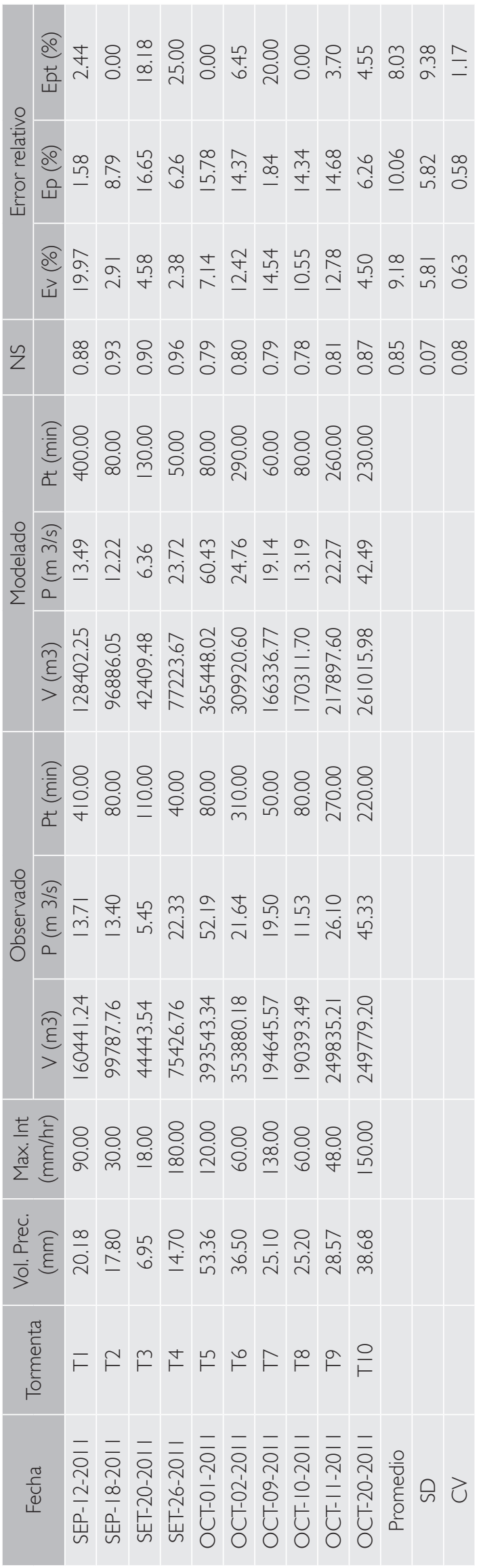

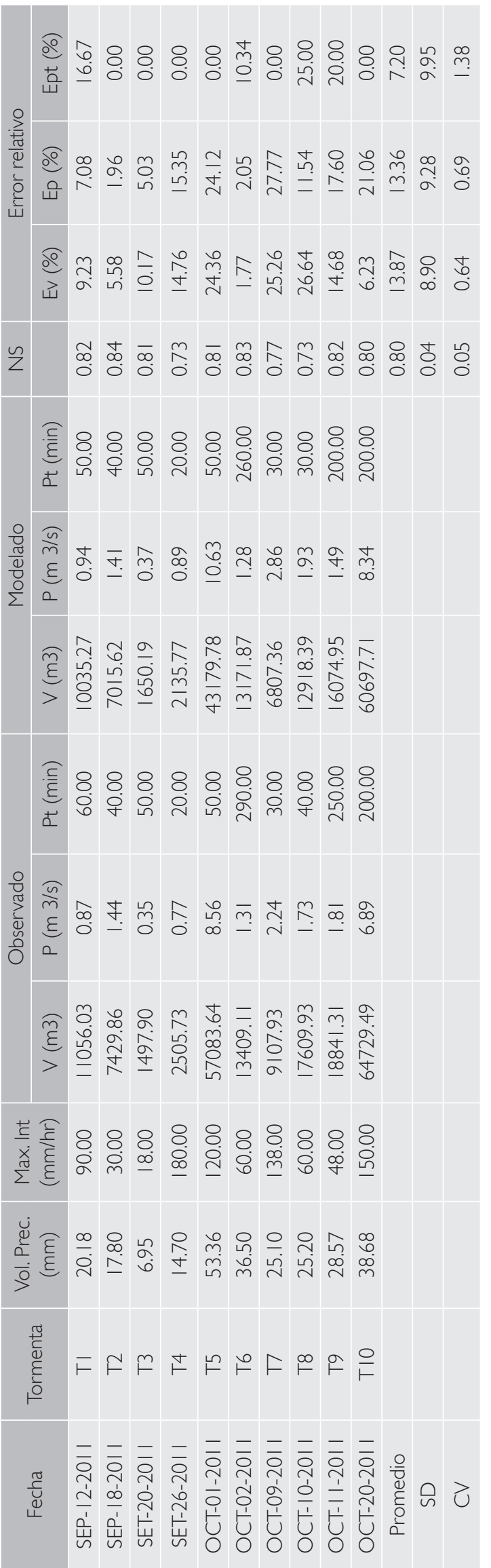


relativo; Ep, el error pico relativo y Ept, el error relativo al tiempo pico.

En ambas cuencas, las intensidades máximas, las cuales corresponden a tormentas ocurridas a finales de septiembre y durante octubre de $201 \mathrm{I}$, alcanzaron valores tan altos como $180 \mathrm{~mm} / \mathrm{hr}$ (tormenta T4) y tan bajos como $18 \mathrm{~mm} / \mathrm{hr}$ (tormenta T3), según muestra la Figura 7. Los volúmenes máximos precipitados van desde los $53.36 \mathrm{~mm}$ (tormenta T5) hasta los $6.95 \mathrm{~mm}$ (tormenta T3). En el caso del río Toyogres, estas tormentas produjeron caudales pico que van desde los $52.19 \mathrm{~m}^{3} / \mathrm{s}$ (tormenta T5) hasta los $5.45 \mathrm{~m} / \mathrm{s}$ (tormenta T3). En el caso de la quebrada Zopilote, de mucha menor extensión, se produjeron caudales pico máximos de $8.56 \mathrm{~m}^{3} / \mathrm{s}$ y mínimos de $0.35 \mathrm{~m}^{3} / \mathrm{s}$ para las mismas tormentas. Lo anterior evidencia una variación de un orden de magnitud, tanto en las intensidades máximas de tormenta como en los caudales pico producidos por estas.

Este espectro tan amplio en la naturaleza de los eventos de tormenta garantiza que en el proceso de calibración de SWMM se tomaron en cuenta condiciones plenamente extremas. Con esto se logra evaluar la robustez de la metodología de calibración paramétrica con PEST y el grado de adaptabilidad del modelo a tales condiciones.

El Cuadro 3 muestra la eficiencia NS de cada tormenta individualmente calibrada con PEST para la cuenca del río Toyogres. Puede apreciarse una eficiencia promedio NS de $0.85 \pm 0.07$, lo cual a su vez representa un CV del 8\%. Rientjes (20I0), en su tratado de modelación hidrológica, establece que un NS de entre 0.6 a 0.8 indica un desempeño del modelo razonablemente bueno, muy bueno entre 0.8 a 0.9 y extremadamente bueno por arriba de 0.9. Es este sentido, una eficiencia promedio de 0.85 sugiere un muy buen desempeño general de SWMM y demuestra que el modelo es capaz de adaptarse a condiciones de iniciación extremas.

La Figura 2 muestra una comparación entre los hidrogramas observados y PEST-calibrados junto con el hietograma correspondiente a la tormenta T9 para la cuenca del río Toyogres. Puede notarse que el patrón de precipitación se extiende principalmente a lo largo de 16 horas, lo cual evidencia una baja intensidad (48 mm/hr, Cuadro 3) contra un volumen precipitado de mediana intensidad $(26.1 \mathrm{~mm}$, Cuadro 3). Para este caso, la eficiencia NS es de 0.8I, mientras que los Ev, Ep y Ept alcanzan valores de I2,78 \%, | 14,68 \% y 3,70\% respectivamente. Un error volumétrico de $12,78 \%$ sugiere un faltante de masa que no se ha introducido adecuadamente en los datos de precipitación del SWMM. Al examinar los datos observados de caudal en la Figura 2, vemos que entre la hora 0:00 y 3:00 existen dos picos del hidrograma general que evidentemente no están siendo modelados por SWMM. La razón parece estar ligada al hecho de que ninguna de las tres estaciones meteorológicas distribuidas en el área de la cuenca fue capaz de registrar los eventos de

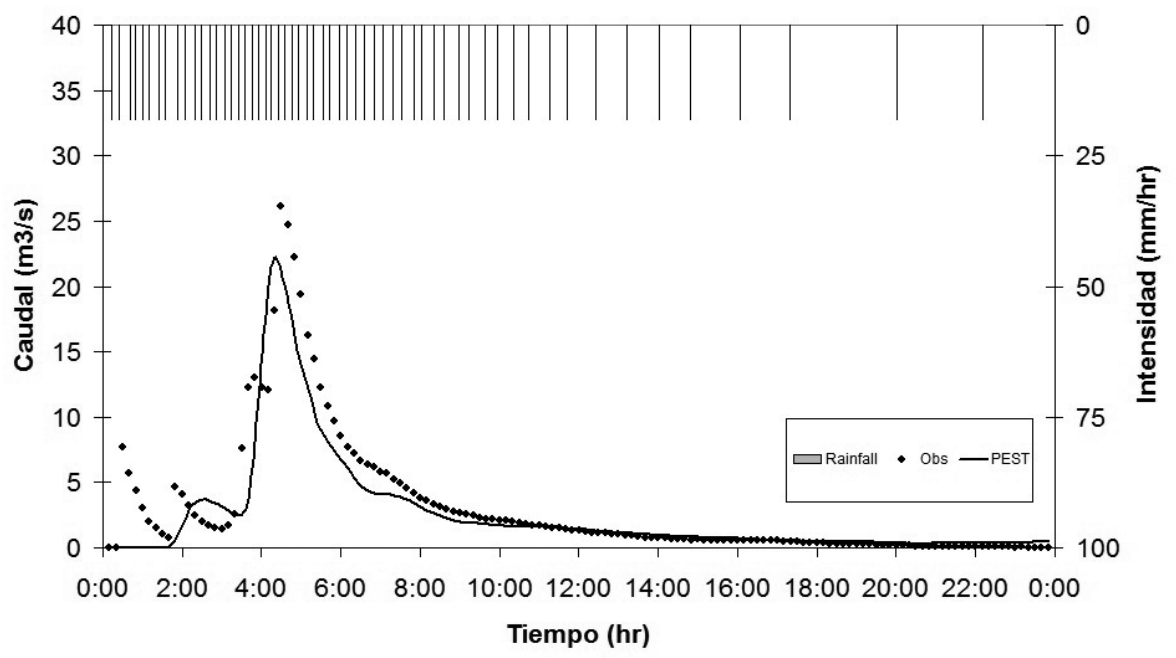

Figura 2. Comparación entre los hidrogramas observados y calibrados junto con el hietograma correspondiente a la tormenta T9 para la cuenca del río Toyogres. 
precipitación que produjeron tales picos. En otras, hubo precipitación localizada sobre alguna sección de la cuenca que no estuvo dentro del área de influencia de ninguna de las estaciones. Este faltante de masa se traduce en una evidente subestimación del caudal pico, por lo que Ep llega a su valor de $14,68 \%$. Esto evidentemente afecta de la misma manera al índice NS, ya que se están comparando valores considerables de caudal observado contra valores modelados de caudal virtualmente iguales a cero.

El cuadro 4 muestra las estadísticas de calibración en el caso de la quebrada Zopilote. La eficiencia general de SWMM sobre esta cuenca es menor que aquella obtenida para la cuenca del río Toyogres, con una eficiencia NS promedio de $0,8 \% \pm 0.4$ y un Ev promedio de $13,87 \% \pm 8.90$, un Ep promedio de 13,36\% \pm 9.28 y un Ept promedio de 7,20\% \pm 9.95, con CV de 0.64, 0.69 y 1.38 respectivamente. Las razones de esta menor eficiencia están directamente ligadas al hecho de que la quebrada Zopilote presenta mucho más infraestructura en relación con el cauce del Toyogres. Mucha de esta infraestructura incluye puentes muy angostos, secciones completas del cauce entubadas e incluso lagunas de retención.

La figura 3, correspondiente a la tormenta T6 en la quebrada Zopilote, muestra cómo el primer pico del hidrograma está considerablemente sobreestimado por parte de SWMM, aun cuando la sincronía temporal es evidentemente admisible. Según SWMM, para las 00:40 debió existir mucho más caudal del que realmente llegó a concentrarse. Lo anterior respalda la hipótesis de que existe retención de flujo por almacenamiento. A manera de contraste, el segundo pico del hidrograma es ligeramente subestimado por SWMM pero sí llega a darse un desfase en el tiempo pico de aproximadamente 20 minutos. Existe también la posibilidad de que exista infraestructura ajena a la caracterización original que pudiera estar aportando flujos externos, correspondientes a otro sector del casco urbano de Cartago.

En cuanto a validación, las eficiencias y errores relativos promedio en ambas cuencas se mantienen muy similares a aquellos obtenidos durante la calibración. En el caso del Toyogres, la eficiencia NS promedio llega a 0.87 , muy similar a la encontrada en calibración con un valor de 0.85. Lo mismo se aplica para la quebrada Zopilote, con un NS en validación de 0.77 contra 0.80 en validación.

Variabilidad de los parámetros

La figura 4 muestra la evolución temporal de los parámetros calibrados por tormenta para la cuenca del río Toyogres. La característica más obvia es el patrón de desplazamiento en los valores de ciertos parámetros, particularmente nper, nimp y ncha, que presentan algunos de los parámetros agregados del modelo SWMM. Sus altos coeficientes de variación (58,75\%, 17,91\% y

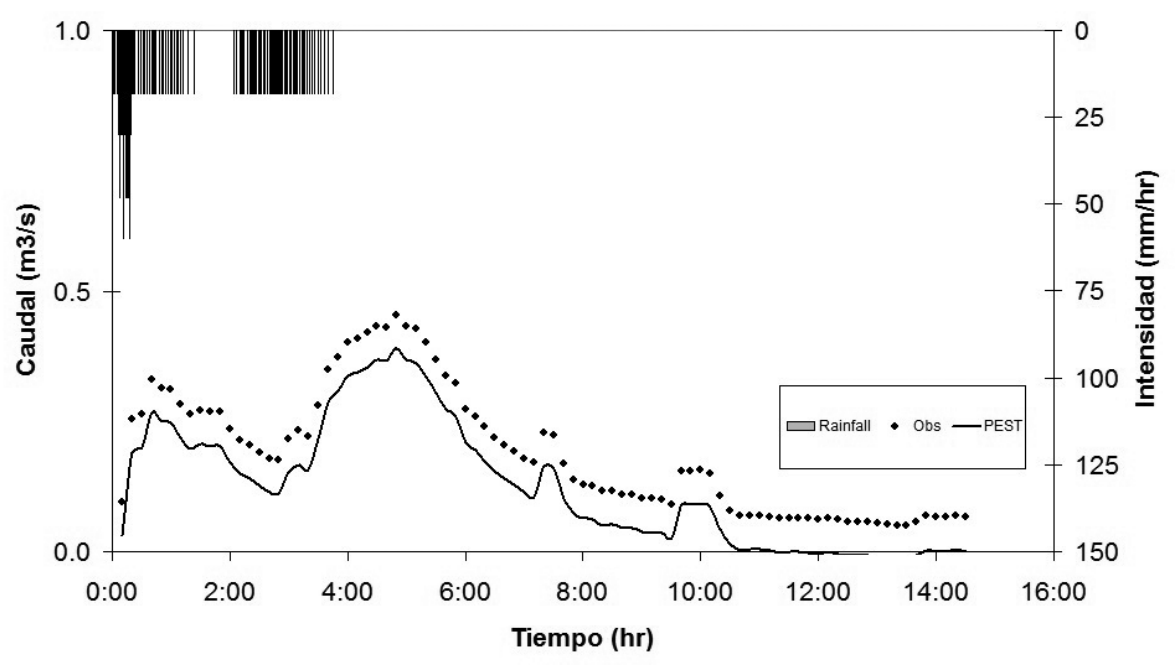

Figura 3. Comparación entre los hidrogramas observados y calibrados junto con el hietograma correspondiente a la tormenta T6 para la microcuenca de la quebrada Zopilote. 


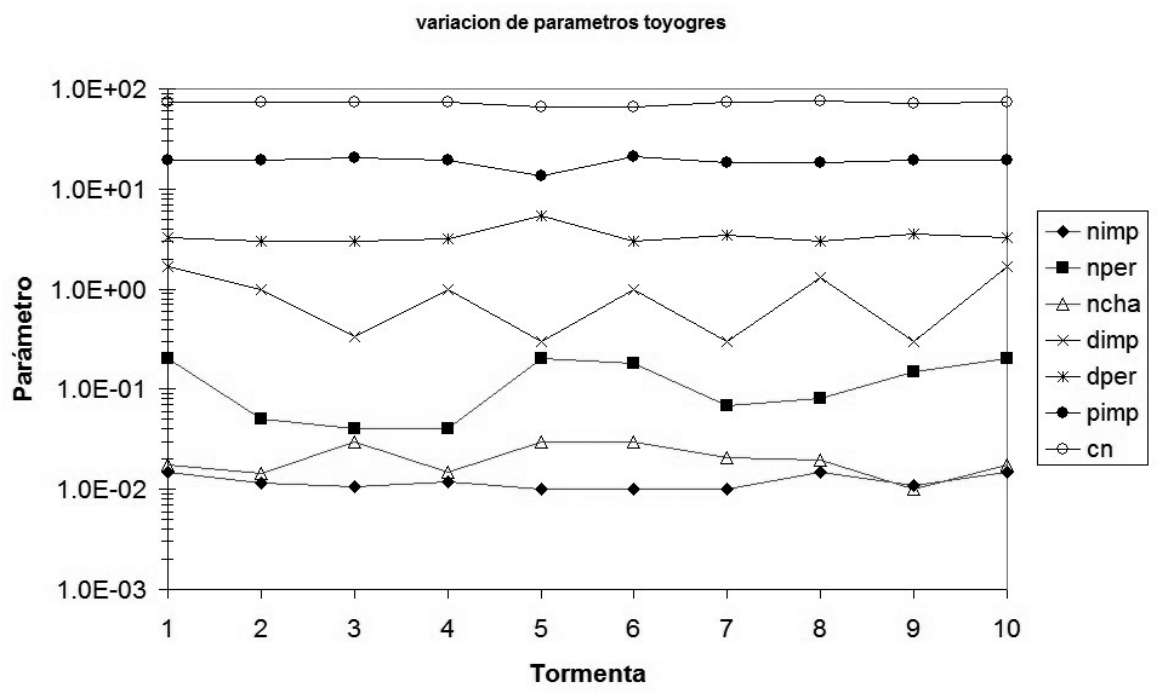

Figura 4.Variabilidad de parámetros calibrados por tormenta para el modelo SWMM en la cuenca del río Toyogres.

$34,87 \%$ respectivamente) subrayan la cambiante naturaleza de tales parámetros. Esto pudiera ser el reflejo de las igualmente cambiantes condiciones que imperan en ambas cuencas y del alto grado de adaptación que presenta SWMM. Lo anterior es de esperar, ya que en el método de calibración utilizado, donde cada tormenta se calibra de forma individual en virtud de observaciones de caudal equivalentemente individuales, la optimización de los parámetros de SWMM por parte de PEST se adapta a aspectos únicos de cada tormenta, lo cual incluye volumen e intensidad. La situación de estos mismos parámetros no resulta diferente en el caso de la cuenca de la quebrada Zopilote, donde los CV alcanzan 33,08\%, I 4,33\% y 17,29\% respectivamente. La variabilidad de las propiedades físicas del suelo, en virtud de su condición de humedad antecedente, es igualmente determinante.

Como consecuencia, la estructura de SWMM tiende a adaptarse a las diferentes relaciones y cocientes entre las entradas y salidas del sistema, sobre todo desde el punto de vista de tránsito hidráulico. En diversos modelos hidrológicos, muchos de los parámetros ligados al tránsito hidráulico, principalmente aquellos relacionados a la rugosidad de las superficies, se consideran parámetros eminentemente físicos y constantes. En este sentido, se debe recordar que la parte alta de la cuenca del Toyogres es eminentemente agrícola, con coberturas vegetales muy diversas y profundos perfiles de suelo; mientras que la microcuenca de la quebrada Zopilote es de carácter meramente urbano, con coberturas vegetales esencialmente ligadas a pastos o charrales menos susceptibles a condiciones de drenaje. Una menor variabilidad de los parámetros en el caso de la quebrada Zopilote versus su menor eficiencia promedio solo puede sugerir que los márgenes de variación de los parámetros, los cuales concuerdan con los del Toyogres, no son suficientes para garantizar una mayor eficiencia del modelo en esta cuenca.

\section{Análisis de sensibilidad y correlaciones}

Estrictamente hablando, la calibración y subsecuente identificación de un parámetro es importante únicamente si el parámetro resulta crítico en la descripción de los procesos que pretende modelar. En consecuencia, la mayor atención en el proceso de calibración debería orientarse hacia los parámetros sensitivos del modelo. De la misma forma, debería procurarse una menor incertidumbre en este tipo de parámetros. En virtud de lo señalado, la Figura 5 muestra la evolución temporal de la sensibilidad relativa de los parámetros calibrados para la cuenca del Toyogres. Lo más llamativo es la disparidad de la sensibilidad entre los grupos de parámetros, que en algunos casos es de varios órdenes de magnitud. Los parámetros ligados a rugosidades de superficie, nimp, ncha y nper resultan los más sensitivos, aunque en distinto orden. El parámetro nimp es levemente más sensible que ncha, más la importancia de ambos está un orden de magnitud por encima de nper. Este 
comportamiento resulta muy similar en el caso del Zopilote, aunque la sensibilidad relativa de nimp es ligeramente superior a ncha. Lo anterior sugiere una vez más el hecho de que PEST mueve a SWMM hasta las bandas superiores en inferiores de sus parámetros ajustables, en un intento de encontrar el set óptimo de parámetros que mejor armonice las observaciones y modelaciones.

En el caso del Toyogres, dimp y dper son mayoritariamente sensibles para las tormentas TI, T2, T9 y TIO. Estas tormentas comparten la particularidad de anteceder o preceder periodos de varios días de muy baja a nula precipitación (cuadro 3), que en el caso de la parte alta de la cuenca del Toyogres podrían igualmente afectar las características de suelo, entre ellas las láminas de almacenamiento, justificando así los picos y valles mostrados por la figura 5. Las sensibilidades por debajo de IE-03 se consideran despreciables y nimp, ncha y nper son, sin duda, los parámetros más sensitivos en ambos modelos de cuencas.

Por otro lado, resulta pretencioso establecer correlaciones para nimp, ncha y nper en ambos modelos de cuenca, todo lo contrario; parecen ser de hecho independientes de la intensidad de precipitación. Como es de esperarse, la dispersión en la cuenca del Toyogres es más alta en virtud de la adaptabili- dad del modelo a sus condiciones de uso de suelo y topología de tormenta.

En el caso del río Toyogres, nimp pareciera mostrar independencia, sí existe una marcada correlación creciente para nper y en menor medida para ncha (Figura 6).

Esto sugiere una vez más la alta dependencia de nper de las condiciones de humedad antecedente y el volumen total precipitado y, por otro lado, respalda lo encontrado por Abrahams et al. (1994), Weltz et al. (1992) y Wu et al. (1999), donde un aumento del tirante efectivo producto de un mayor volumen escurrido conlleva una mayor rugosidad compuesta del cauce del río, principalmente como consecuencia de la contribución de la vegetación sobre la rugosidad compuesta de la sección.

\section{Conclusiones}

Se aplicó el modelo hidrológico SWMM a un caso de estudio que incluyó el análisis de dos cuencas hidrológicas en el área urbana de la ciudad de Cartago, Costa Rica. Para la optimización de parámetros, calibración y análisis de sensibilidad de SWMM, se utilizó la suite de calibración y optimización paramétrica PEST, la cual se fundamenta en los algoritmos de Gauss-Marquardt-Levenberg (GML).

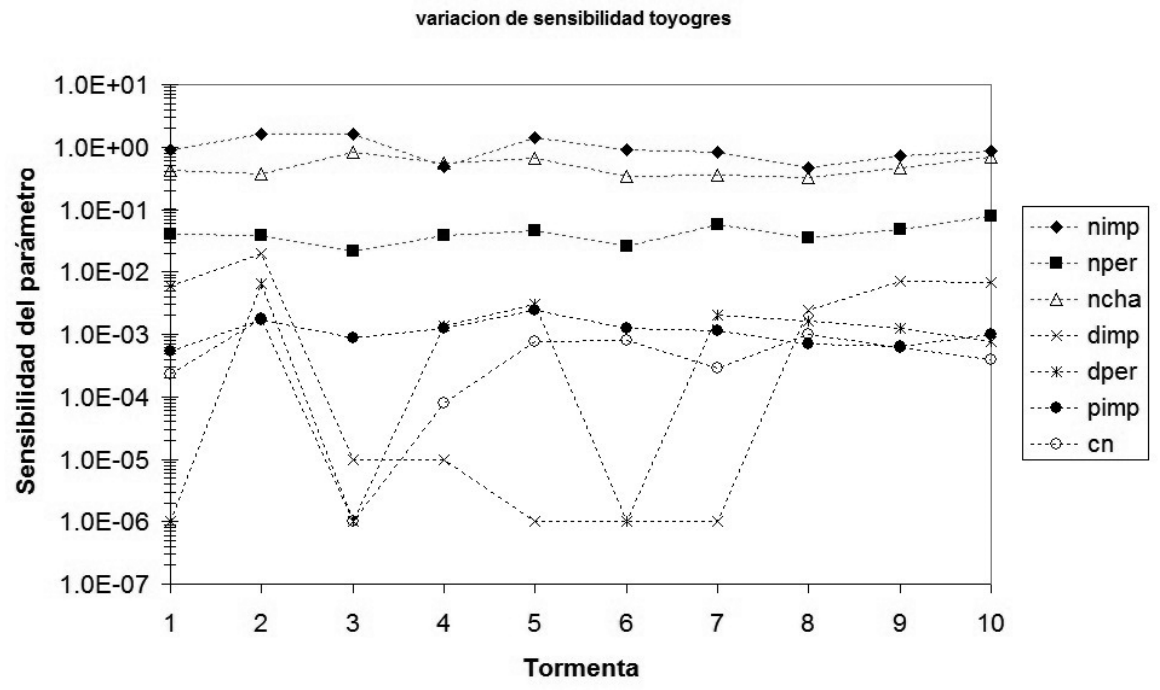

Figura 5. Variabilidad de la sensibilidad de los parámetros calibrados por tormenta para el modelo SWMM en la cuenca del río Toyogres. 


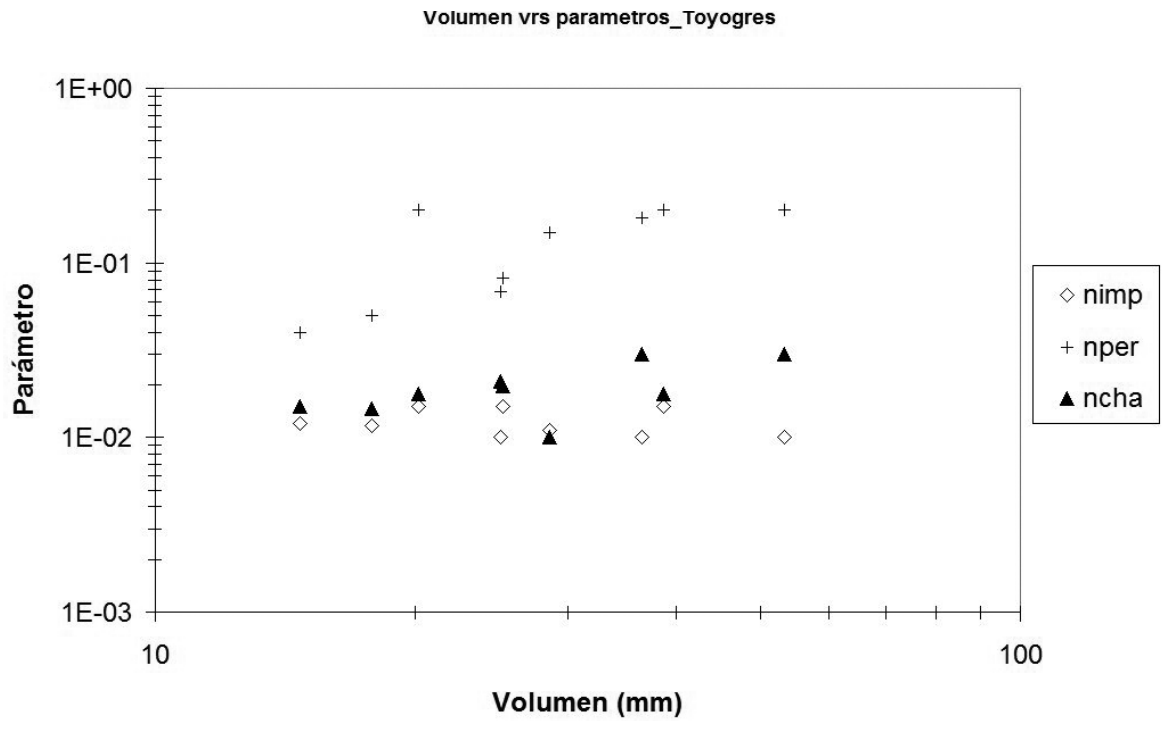

Figura 6. Variación de parámetros del modelo SWMM en función del volumen de precipitación por tormenta para el modelo SWMM en la cuenca del río Toyogres.

En el proceso de calibración con PEST se utilizaron 10 eventos secuenciales de tormenta registrados por tres estaciones meteorológicas durante la estación lluviosa del año 201 I. La evolución de los parámetros, junto con su sensibilidad relativa, fue analizada para cada evento seleccionado haciendo uso de los hidrogramas observados. El análisis de dichos eventos indica que tanto los parámetros como las sensibilidades relativas $y$, por ende su importancia, cambian en relación con la tipología de cada tormenta y con el régimen de precipitación del año hidrológico, lo cual a su vez indica que no existe un único set óptimo de parámetros que se adapte satisfactoriamente a todas las condiciones.

Los coeficientes de rugosidad de Manning para el flujo superficial sobre el área impermeable (nimp), el flujo superficial sobre el área permeable (nper) y el tránsito hidráulico (ncha) resultaron los parámetros más sensitivos de todos, siendo estos últimos de carácter totalitario. El comportamiento de estos parámetros es parcialmente dependiente de la intensidad y volumen total de precipitación en relación con los usos y coberturas que dominan el espacio de cada cuenca analizada. Su comportamiento también sugiere que existe una profunda dependencia con respecto al estado de humedad antecedente del suelo y los estados de flujo hidráulico, tanto a nivel de superficie como de tránsito hidráulico.
Aun cuando los coeficientes de variación de estos parámetros son relativamente altos, SWMM demostró ser lo suficientemente flexible para adaptarse satisfactoriamente a todas las tipologías de tormenta analizadas, dado que las eficiencias medias NS en calibración oscilan entre 0.85 y 0.80 , mientras que en validación oscilan entre 0.87 y 0.77 para las cuencas del Toyogres y el Zopilote respectivamente. De la misma manera, los errores volumétricos, de caudal pico y tiempo pico no superan el I5\% en ninguno de los casos.

Lo anterior es de suma importancia, ya que si bien es cierto los parámetros sensitivos de SWMM no son enteramente físicos y varían en bandas relativamente anchas, demuestran que el modelo se puede utilizar incluso en condiciones donde no existen datos observados para su calibración o validación dada la alta flexibilidad del modelo hidráulico de onda cinemática de SWMM. No debe dejarse de lado el hecho de que las observaciones de caudal no están exentas de errores, ya que su deducción depende de una medida indirecta de velocidad y nivel piezométrico.

Los parámetros distribuidos tomados en cuenta durante la calibración inicial con PEST exhibieron sensibilidades con órdenes de magnitud más bajas que aquellas presentadas por los coeficientes de rugosidad. Esto demuestra que la calidad de los datos espaciales fue la adecuada en la parametriza- 
ción de SWMM, en el tanto que se logra dar una interpretación razonablemente física de tales parámetros distribuidos.

\section{Bibliografía}

Abrahams, A.D., Parsons, A.J. \& Wainwright, J. ( 1994). Resistance to overland flow on semiarid grassland and shrubland hillslopes, Walnut Gulch, southern Arizona. Journal of Hydrology I 56 ( I-4), 43 I-446.

Barco, J., Wong, K.M. \& Stenstrom, M.K. (2008). Automatic calibration of the U.S. EPA SWMM model for a large urban catchment. ASCE J. Hydraul. Eng. 34I (3-4), 207-22I.

Barredo, J. (1996). Sistemas de información geográfica y evaluación multicriterio. Madrid: RA-MA Editorial.

Boyko, O. \& Treebushny, D. (2006). Application of a physically based distributed rainfall-runoff model (TOPKAPI-IMMSP) to gauged and ungauged mountainous catchments of ranscarpathean region, Ukraine. Geophys Res. Abstr 8: 07964.

Chow, V.T., Maidment, D.R. \& Mays, L.W. (1988). Applied Hydrology. New York: McGraw Hill.

Doherty, J. (2005). PEST: model independent parameter estimation. User's Manual. Watermark Numerical Computing, Brisbane.

Gallagher, M.R. \& Doherty, J. (2007). Parameter interdependence and uncertainty induced by lumping in a hydrologic model. Wat. Res. Res. 43 (5), I - I8.

Gupta, V.K. \& Sorooshian, S. (1985). The relationship between data and the precision of parameter estimates of hydrological models. J. Hydrol. 81, 57-77.

Hogue, T., Gupta, H. \& Sorooshianc, S. (2006). A User-friendly approach to parameter estimation in hydrologic models. J. Hydrol. 320 ( I-2), 202-2 I7.

Hsu, M.H., Chen, S.H. \& Chang, T.J. (2000). Inundation simulation for urban drainage basin with storm sewer system. J. Hydrol. 234 (I-2), 21-37.

Huber, W. \& Dickinson, R. (1988). Storm Water Management Model Version 4, Part A: User's Manual. EPAI60013-88100 Ia. US Environmental Protection Agency.
Jain, M.K., Kothyari, U.C. \& Ranga Raju, K.G. (2004). A GIS-based distributed rainfall-runoff model. Journal of Hydrology 299 (I-2), | $07-135$

Kumar, S.M., Narasimhan, S. \& Bhallamudi, S.M. (2009). Parameter Estimation in Water Distribution Networks. J. Wat. Res. Mngmnt. 24 (6), | 25 |- 1272.

Maneta, M.P., Pasternack, G.B., Wallender, W.W., Jetten, V. \& Schnabel, S. (2007). Temporal instability of parameters in an event-based distributed hydrologic model applied to a small semiarid catchment. Journal of Hydrology 34I, 207- 221.

Marcel van der Perk, O.S. (2003). Simulation of event-based and long-term spatial redistribution of Chernobyl-derived radiocae-sium within catchments using geographical information system embedded models. Hydrol. Process. 17 (5), 943-957.

Mays, L.W. (2000). Water distribution systems handbook. New York: McGraw-Hill.

Nash, J.E. \& Sutcliffe, J.V. (1970). River flow forecasting through conceptual models part I $\mathrm{d}$ a discussion of principles. Journal of Hydrology $10(3), 282-290$

Rientjes, T.H.M. (20I0). Modelling in Hydrology. Department of Water Resources. Fc. Of Geo-Information Science and Earth Observation. University of Twente.

Tsihrintzis, V. \& Hamid, R. (1998). Runoff quality prediction from small urban catchments using SWMM. Hydrol Process I2(2), 31 |-329.

Skahill, B.E. (2009). More efficient PEST compatible model independent model calibration. J. Env. Model. Softw. 24 5 I7-529.

Veihe, A., Rey, J., Quinton, J.N., Strauss, P., Sancho, F.M. \& Somarriba, M. (200I). Modelling of event-based soil erosion in Costa Rica, Nicaragua and Mexico: evaluation of the EUROSEM model. CATENA 44(3), I87--03.

Wanielista, M.P. \& Youssef,Y.A. ( 1 993). Stormwater Management. New York: John Wiley \& Sons, Inc.

Weltz, M.A., Arslan, A.B. \& Lane, L.J. (1992). Hydraulic roughness coefficients for native rangelands. Journal of Irrigation and Drainage Engineering I | 8(5), 776-790.

Wu, F.C., Shen, H.W. \& Chou, Y.J. (1999). Variation of roughness coefficients for unsubmerged and submerged vegetation. Journal of Hydraulic Engineering, ASCE I25(9), 934-942. 